\title{
Apropiación tecnológica y cuerpos femeninos: el camino del código abierto
}

\author{
Martha Irene Soria Guzmán ${ }^{1}$ \\ Universidad del Claustro de Sor Juana
}

\section{RESUMEN}

En muchos casos, la apropiación tecnológica se ha asociado al manejo de herramientas o dispositivos que a su vez han sido mediados por corporativos que los usan como mecanismos de control. De igual forma, el sistema heteropatriarcal ha normalizado la dominación del cuerpo femenino a través de ideas sesgadas de lo que significa "ser mujer".

En ambos casos, los posibles vehículos para la emancipación podrían ser: el conocimiento, la confianza y la capacidad de decisión sobre nuestra tecnología y nuestros cuerpos.

El presente ensayo, propone que la apropiación tecnológica vaya más allá del manejo de una herramienta, ya que es preciso saber cómo fue hecha, conocerla, intervenirla o crear una propia, lo cual, solo será posible con software libre, cuya filosofía de código abierto, compartición y colaboración en beneficio de la comunidad, coinciden con la filosofía backer y el feminismo.

Palabras clave: software libre, open source, código abierto, feminismo, mujeres, hacker

\section{ABSTRACT}

In many cases, technological appropriation has been associated to the management of tools or devices that in turn have been mediated by corporations that use them as control mechanisms. Similarly, the heteropatriarcal system has normalized the domination of the female body through biased ideas of what it means "be a woman". In both cases, the possible vehicles for emancipation will be: knowledge, trust and the ability to decide on our technology and our bodies.

This academic essay proposes that the technological appropriation goes beyond the management of a tool, since it is necessary to know how it was done and create it of its own, which will only be possible with free software, whose

${ }^{1}$ Candidata a doctora en Estudios Transdiciplinarios de Comunicación y Cultura. Profesora-investigadora del Colegio de Comunicación de la Universidad del Claustro de Sor Juana. Miembro del Consejo Consultivo de la Fábrica Digital "El Rule" en la CDMX. Correo electrónico: isoriag@elclaustro.edu.mx 
philosophy of open source, sharing and collaboration for the benefit of the community, is similar to the hacker philosophy and some feminisms.

Key words: free software, open source, open code, feminism, women, hacker 


\section{INTRODUCCIÓN}

Los equipos de cómputo se han convertido en herramientas tecnológicas presentes en algunos aspectos de la vida cotidiana urbana. El uso de computadoras de escritorio o equipos móviles y portátiles en la escuela, el hogar y la oficina, se ha vuelto más común, natural e indispensable, siendo este hecho para algunos, una muestra de la innovación y progreso de nuestra era, así como la oportunidad de adquirir nuevas habilidades (Villanueva, 2010).

En este sentido, se confunde el término apropiación tecnológica con el uso instrumental de gadgets o dispositivos móviles tales como smartphones, tablets o laptops, ya que el acercamiento y conocimiento de la tecnología se ha reducido al uso de aplicaciones que agilizan alguna actividad de las personas que lo usan o que simplemente, las entretienen. Sin embargo, sabemos que las empresas fabricantes de estos gadgets, dispositivos, software y aplicaciones, son mediadores de la interacción con los usuarios y que sus intenciones no siempre son claras, incluso, se ha probado que algunas de estas prácticas de mediación son invasivas para la privacidad de miles de consumidores finales.

De manera paralela, la significación del cuerpo femenino ha sido transmitida a través de múltiples mediaciones, entre las que se en-
Martha Irene Soria Guzmán cuentran los medios masivos de comunicación, la literatura, el cine y el arte. Éstos nos han ofrecido por años una idea sesgada de lo que significa "ser mujer", pero también lo que significa "ser hombre", marcando ciertas "pautas" de comportamiento y "deber ser" hacia ambos sexos, e incluso, ciñendo la identidad sexual a un "binomio", dejando en la "anormalidad" todo lo que no se suscriba a la categoría hombre o mujer (Mathieu, 2005). Sin embargo, aunque es relativamente clara la imposición mediática de roles en ambos casos, es de particular interés de este ensayo, la reflexión únicamente en torno al cuerpo de las mujeres, dado que, como apunta Bourdieu (1998, p. 22) "el orden social funciona como una inmensa máquina simbólica que tiende a ratificar la dominación masculina, en la que se apoya: la división sexual del trabajo, distribución muy estricta de las actividades asignadas a cada uno de los dos sexos, de su espacio, su momento, sus instrumentos...", siendo las mujeres objeto de esa dominación.

Este ensayo plantea una coyuntura entre la mediatización de la tecnología y la mediación en la representación del cuerpo femenino. Propone a su vez, acercarse a la filosofía del software libre, en la que se conoce, estudia, mejora y modifica el código a partir de un trabajo en comunidad; en contraposición a la 
filosofía "privativa" o de código cerrado que impera en la industria, donde solo un pequeño grupo de personas puede acceder a él.

Con esta propuesta, se busca establecer un planteamiento donde el conocimiento, la confianza y la capacidad de decisión sobre nuestras herramientas digitales y sobre nuestros propios cuerpos, nos acerque a una verdadera apropiación, tanto tecnológica como del ser femenino.

\section{CONTROL MEDIÁTICO: EL USO DE} LA HERRAMIENTA TECNOLÓGICA

Año con año, se ha incrementado el uso de dispositivos móviles en el mundo. En el caso de México y particularmente en su sector educativo, es interesante observar que para el ciclo escolar 2014-2015, la Secretaría de Educación Pública entregó 709 mil 824 tabletas electrónicas a 664 mil 201 alumnos y alumnas de quinto grado de educación primaria y a 45 mil 623 profesores y profesoras de escuelas de varios Estados de la República mexicana (Notimex, 2014), gracias al Programa de Inclusión y Alfabetización Digital, bajo la premisa de que usar las tecnologías de la información y comunicación es beneficioso para el aprendizaje. Del 2012 al 2015, se han invertido tres mil 763 millones 869 mil 669 pesos para adquirir 240 mil laptops y un millón 669 mil 864
Martha Irene Soria Guzmán tabletas para niños de escuelas públicas (Ascención, 2016).

Además de que estos productos se han vuelto auxiliares en gran parte de las actividades humanas, también poseen una especie de valor "agregado", que los vuelve símbolos sociales de estatus y poder. Para algunos jóvenes es más importante la posesión de un aparato de telefonía celular que el uso que se le otorga; de igual forma, "para los hombres el mostrar el celular puede llegar a ser un símbolo de estatus y virilidad, o en el caso de las mujeres, un 'no estoy sola, estoy con mi celular"' (Castañeda, 2010, p.62).

Estos gadgets y/o aparatos tecnológicos se convierten en objetos de consumo de los cuales desaparece su función objetiva "útil", transformándolos en un signo con una inutilidad funcional. Estos signos, dentro de la sociedad de consumo "jerarquizan a los grupos sociales manteniendo su estructura de desigualdad y dominio" (Braudillard, 2009, p. 130).

Según las citas anteriores, en la sociedad de consumo estos aparatos además de vender "estatus", favorecen la desigualdad y diferencia de clases entre quienes buscan su identidad en estos signos provenientes de la industria. El objeto se vuelve un amuleto de culto, mágico e inalcanzable, mientras que las personas 
que lo usan son consumidores cautivos a expensas de las nuevas versiones de los aparatos, los servicios técnicos de las empresas fabricantes o la durabilidad de los mismos.

Una de las marcas icónicas en la producción de tecnología y que desde sus orígenes estuvo orientada a mostrar una imagen de innovación e exclusividad, fue Apple $囚$. Una de las consignas de esta marca no es solo vender equipos de cómputo, sino vender la idea de sofisticación al poseer una Mac ${ }^{\circledR}$. Hoy en día, esta estrategia sigue siendo la bandera de Apple, ya que como lo menciona la columnista del blog del periódico El País: “...es surrealista hacer un seguimiento en vivo del lanzamiento de un producto de una empresa privada cuando no se hace con ninguna otra"(Rodríguez, 2011).

Según uno de los vendedores ejecutivos de la marca, Apple no vende "productos" sino un estilo de vida: "...te está invitando a experimentar el estilo de vida Apple y ser parte de la comunidad $\mathrm{iPod} \AA$...Los auriculares del iPod, por ejemplo, son un puro truco de marketing diseñados para que la única parte visible del producto sea un símbolo de estatus: usar blanco" (Chazin, 2011). En este sentido, Apple ha sido una de las marcas tecnológicas que más ha representado la idea de estatus en
Martha Irene Soria Guzmán este sector y que incluso marca al consumidor como miembro de un grupo selecto.

Sin embargo, Apple y otros tantos corporativos no solo fabrican y distribuyen estos aparatos tecnológicos en cuanto a sus componentes físicos o hardware, sino que también producen el software que los hace funcionar, lo que añade otro mecanismo poco conocido que ha sido usado también para el control.

En este sentido, Richard Stallman, padre del software libre, dice que el software es un conjunto de recetas minuciosamente detalladas para la solución de un determinado problema, que puede ir desde hacer una suma hasta escribir una carta, crear un vector o editar un video. Dichas recetas están escritas de una manera muy parecida a como la música se escribe, usando notaciones propias. A esto podemos llamarle lenguajes formales que son con los que están escritos dichos programas (Busaniche, 2012).

Así pues un software o programa de computadora puede ser escrito por cualquier persona que conozca alguno de estos lenguajes formales, mejor conocidos como lenguajes de programación, de la misma manera en la que cualquiera puede escribir una novela en un idioma diferente al español, siempre y cuando se conozcan sus reglas gramaticales. 
Richard Stallman dice en su libro Software libre para una comunidad libre (2004), que cuando el software como producto comercial estaba aún lejano, cada programa se creaba para cada hardware y su distribución generalmente se quedaba dentro de la empresa donde un equipo de programadores lo corregiría en caso de tener errores, o bien, lo adaptaría a nuevas necesidades. Incluso, era muy común compartir programas entre los programadores y con ello, pedir parte del código fuente ${ }^{2}$ para mejorarlo entre todos: "El acto de compartir software no se circunscribe a nuestra comunidad en particular: es tan antiguo como los propios ordenadores, lo mismo que compartir recetas es tan viejo como la cocina" (Stallman, 2004, p. 9).

Sin embargo, en los años ochenta algunas empresas pioneras de computación comenzaron a contratar cientos de programadores para crear programas que no pudieran compartirse y fueran utilizados solo por la empresa. Algunas computadoras modernas de la época co-

${ }^{2} \mathrm{El}$ código fuente es un conjunto de líneas de texto que son las instrucciones que debe seguir la computadora para ejecutar dicho programa. Por tanto, en el código fuente de un programa está descrito por completo su funcionamiento y está escrito en algún lenguaje de programación, pero en este primer estado no es directamente ejecutable por la computadora, sino que debe ser traducido a otro lenguaje (el lenguaje máquina) que sí pueda ser ejecutado por el hardware de la computadora. A partir de ahora nos referiremos al código fuente solo con la palabra "código".
Martha Irene Soria Guzmán menzaban a tener su propio sistema operativo (SO) para el cual, en ocasiones se necesitaba firmar un acuerdo de confidencialidad para obtener una copia ejecutable:

Todo ello significaba que antes de poder utilizar un ordenador tenías que prometer no ayudar a tu vecino. Quedaban así prohibidas las comunidades cooperativas. Los titulares de software propietario establecieron la siguiente norma: "Si compartes con tu vecino, te conviertes en un pirata. Si quieres hacer algún cambio, tendrás que rogárnoslo" (Stallman, 2004, p. 21).

Poco a poco, la cultura del uso y compartición del código que predominaba en los años setenta, se transformó paulatinamente en su "privatización", volviéndolo cerrado y desembocando luego en la creación y uso de patentes. Muy poco tiempo después, la venta de software se volvió un negocio muy redituable para las corporaciones.

A partir de entonces comienza la era de la comercialización de un software que no tenía el código fuente abierto para que todos los usuarios lo conocieran y estudiaran, por el contrario, se trataba de un código cerrado que representó un velo que impediría saber cómo fue hecho, es decir, imposibilita al usuario final que conozca la receta con la que fue creado el 
programa. Comienza la era del uso de una "caja negra" como herramienta tecnológica (Soria, 2012). Cabe señalar que hoy en día al adquirir estos programas, se paga una licencia que autorice el uso legal de ese software y no el programa en sí mismo. En pocas palabras, se paga el permiso para usarlo.

Esta imposibilidad de ver y modificar el código, vulnera fuertemente al usuario final, convirtiéndolo en un consumidor cautivo que está a expensas de las nuevas actualizaciones y de programas compatibles con su hardware o con sus necesidades.

Ejemplo de esto son las comunidades de profesionales del diseño o de la comunicación, quienes supeditan su actividad profesional al uso de software de patente que controla gran parte de su quehacer profesional. Ellos se enfrentan al cambio de versiones de los programas, a la interoperabilidad de los mismos e incluso a no poder abrir un archivo hecho con una versión antigua del software, impidiendo la conservación digital de sus obras en el tiempo.

Pero la imposibilidad de ver la receta de cocina y el uso generalizado de una caja negra no solo trae como consecuencia la generación de público cautivo, sino que además representa otro tipo de control aún más contundente y evidente que tiene que ver con el uso de datos personales de los usuarios. Gracias a las decla- raciones del ex-contratista de la NSA estadounidense (National Security Agency) Edward Snowden, hemos sabido que ciertos servicios en línea y programas de patente, incluyendo sistemas operativos, se valen de las actualizaciones de sus diferentes versiones para implementar "código espía" que sirve a terceros para monitorear las preferencias, movimientos y actividades de los usuarios con fines inciertos, presuntamente publicitarios disfrazados de "mejoras" en la experiencia de usuario; en muchas de estas ocasiones, el usuario no se entera que esto atenta severamente su privacidad, aunque lo permite al aceptar los términos uso. Cabe señalar, que no aceptar los términos de uso impide el correcto funcionamiento de ciertos aparatos o servicios, incluso, lo imposibilitan, es decir, no hay más opción para los usuarios finales que dar clic al "sí" en los términos de uso o no acceder al aparato o servicio (Ricaurte, et. al., 2014).

Éstas y otras implicaciones han hecho que un grupo de personas opten por llamarle software privativo a este software para subrayar la definición de un software que "priva" libertades y ofrece ciertas limitaciones. En este sentido, cabe hacer una aclaración que, si bien el término software propietario se ha usado para referirse a este software de patente, el propio Richard Stallman, comenzó a utilizar en 
2003 el término software privativo, ${ }^{3}$ acuñado por el activista argentino Enrique Chaparro. $\mathrm{Al}$ respecto, Stallman dice:

...teníamos el término 'propietario', pero hace tres años más o menos, Enrique Chaparro, un argentino, activista del software libre, pensó que la palabra 'propietario' es un nombre y no un adjetivo, ${ }^{4}$ y que, en consecuencia, 'privativo' expresa más claramente el mal que hay en este software: el mal está en privar de libertad a los usuarios. Por eso decimos ahora software privativo, aunque algunos sigan llamándolo propietario como nosotros lo decíamos hace unos años (Morán, 2006).

En este mismo sentido, algunas personas han decidido crear y optar por el camino del software libre, el cual permite no sólo que los usuarios conozcan las líneas de código con el que se ha hecho el programa, sino que sea copiado, distribuido y mejorado sin restricción ni problema legal alguno, convirtiéndose así en una alternativa para conocer el interior de

3Para fines de este ensayo académico, es conveniente el uso del término software privativo, dado que el término software propietario como contraparte al termino software libre, podría dar la idea de que los programas libres no tienen "dueño" o "autor", lo cual es incorrecto. Las licencias de software libre respetan la autoría y como tal, se publican en la propia licencia del software.

"Según la RAE, "propietario" sí es un adjetivo, pero no denota privación de libertad.
Martha Irene Soria Guzmán

una caja negra. Pero de esto y las alternativas para la emancipación de este contundente control corporativo, hablaremos más adelante.

\section{CONTROL MEDIÁTICO: CÓMO HAY QUE SER MUJER}

La noción de lo femenino ha sido definida en múltiples manifestaciones culturales a lo largo de la historia de la humanidad. La religión, la literatura, la escultura, la pintura, y más recientemente, el cine, la publicidad y los medios masivos de comunicación, han interpretado y transmitido a la sociedad el "deber ser" femenino. Estas definiciones varían, por supuesto según la época y la geografía, el contexto cultural, las condiciones socio-políticas, etcétera.

Sin embargo, podemos encontrar algunas similitudes e ideas generalizadas de la femineidad que se han perpetuado a lo largo de los años, algunas de ellas, han tratado de ser transcendidas por las propias mujeres en diversas épocas, pero otras más aún son asumidas como estigmas en pleno siglo XXI. Por supuesto, muchas de estas definiciones surgen desde lo masculino y desde una sociedad dominada por la visión patriarcal.

Por poner solo un ejemplo, en el libro Cultura femenina, editado en español en 1934 pero escrito en Alemania en la primera década del siglo XX, su autor, Jorge Simmel, hace re- 
ferencia a la femineidad como objeto de sometimiento a medidas y necesidades de origen masculino:

La feminidad no puede nunca ser juzgada por normas propias. De esta suerte, la mujer queda sometida al mismo tiempo a dos medidas distintas y ambas de origen masculino; una es la medida absoluta — formada por los criterios de los hombres - que se aplica a las actividades de la mujer, a sus convicciones, a los contenidos teoréticos y prácticos de su vida; otra es la medida relativa, que también procede de la prerrogativa del hombre y que muchas veces formula exigencias totalmente opuestas. El hombre exige de la mujer no sólo lo que le parece deseable en general, sino también lo que le parece deseable como hombre, como término aislado y contrapuesto a la mujer: exige de ella la feminidad en el sentido tradicional de la palabra que no significa un modo de ser peculiar, con su centro propio, sino una índole especial, orientada hacia el varón, para agradarte, servirle y completarle (Simmel, 1934, p. 88).

Esta idea de lo femenino orientada al varón, responde también a la "virtud" de asumirse como autoridad que "controla" de manera objetiva el devenir de las familias y expresiones colectivas justificando la transformación de la fuerza en derecho:
Martha Irene Soria Guzmán

Mas el dominio del varón sobre la hembra sirve también de fundamento a otra tendencia que conduce a idénticos resultados. Toda dominación fundada en la prepotencia subjetiva ha intentado siempre procurarse una base objetiva, esto es, transformar la fuerza en derecho. La historia de la política, del sacerdocio, de las constituciones económicas, del derecho familiar, está llena de ejemplos, Sí la voluntad del pater familias impuesta a la casa aparece revestida con el manto de la "autoridad", ya no es posible considerarla como explotación caprichosa de la fuerza, sino como expresión de una legalidad objetiva, orientada hacia los intereses generales, impersonales, de la familia (Simmel, 1934, p. 87).

Desde este punto de vista, toda imposición o dominación por parte de la figura masculina se vuelve lógica, pues está basada en la verdad y exactitud objetivas, validando así, una especie de superioridad:

Según esta analogía, y a veces en esta misma conexión, la superioridad psicológica de las manifestaciones masculinas sobre las femeninas, en virtud de la relación de dominio entre el hombre y la mujer, se convierte en una superioridad, por decirlo así, lógica. Lo que el hombre hace, dice y piensa, aspira a tener la significación de una norma, porque revela la verdad y exactitud obje- 
tivas, válidas por igual para todos, hombres y mujeres (Simmel, 1934, p. 87)

De acuerdo con este planteamiento, la femineidad ha sido definida desde el punto de vista del "otro", a través de los ojos del otro y para ser más precisos, desde el "otro" ojo masculino; por lo tanto, muchas de las imposiciones y mecanismos de control entorno a lo femenino vienen de 'fuera' del propio cuerpo, de normas impuestas por una autoridad validada y legalizada por sí misma.

Lo anterior, además inscrito en torno a instituciones que "moldean" el cuerpo (tanto de hombres como de mujeres) como un producto social dentro de relaciones de poder $y$ dominación, como dice Foucault (1974, p. 125), "Ha habido, en el curso de la edad clásica, todo un descubrimiento del cuerpo como objeto y blanco de poder. Podrían encontrarse fácilmente signos de esta gran atención dedicada entonces al cuerpo, al cuerpo que se manipula, al que se da forma, que se educa, que obedece, que responde..."

Actualmente, estas ideas de poder y dominación se ven reflejados en los medios de comunicación y en la publicidad, causando una desconexión de la mujer con su cuerpo gracias a la masificación de los estereotipos. La representación femenina en diversos me- dios, sobre todo a partir del boom de la publicidad a finales del siglo XIX ha sido la de mujer joven, sin arrugas, sin imperfecciones, de buena apariencia, casi inhumana y siempre aludiendo a la belleza y al sexo (Bernad, 2015).

Una vez más, esta idea de la mujer está construida desde y para un público masculino, la cual se vincula fuertemente con la idea del sexo y dominación, donde los hombres pueden adquirir ciertos atributos al consumir dichos productos con el fin de conseguir mujeres como si se tratasen de objetos. De igual forma, la figura del cuerpo femenino que hasta el siglo XIX era vetada y prohibida, ahora se libera para ser mostrada y vender:

Muchas de las campañas publicitarias de la industria vinculada con la belleza en donde prima el culto al cuerpo (moda, perfumes, salud, cosmética, nutrición, joyería, deporte etc.) así como la industria que se dirige más al público masculino (alcohol, cerveza, perfumes y coches) está estrechamente hermanada con el sexo. $\mathrm{Y}$ es que en el siglo XIX, con el auge de la economía y de las clases medias en las ciudades, es cuando se produce un desbordamiento de la demanda por la oferta de productos y es entonces cuando el sistema capitalista necesitó "impulsores del consumo" y la utilización del sexo, a través de mostrar el cuerpo femenino entonces vetado, fue una ca- 
tapulta que cautivó la atención sobre los productos que la utilizaban (Bernad, 2015, p. 2579).

Súbitamente, este "redescubrimiento" del cuerpo bajo la idea de liberación sucede luego de un velo de "prohibición" y una era de puritanismo, tal como lo menciona Baudrillard:

Este "redescubrimiento" que, bajo el signo de la liberación física y sexual, se produce después de una era milenaria de puritanismo, su omnipresencia (y específicamente del cuerpo femenino, habrá que ver por qué) en la publicidad, en la moda, en la cultura de masas —el culto higiénico, dietético, terapéutico de que se lo rodea, la obsesión de juventud, de elegancia de virilidad/feminidad, los tratamientos de belleza, los regímenes, las prácticas sacrificiales asociadas a él, el Mito del Placer que lo envuelve- son todos testimonios de que el cuerpo hoy ha llegado a ser objeto de salvación. Ha sustituido literalmente al alma en su función moral e ideológica (Baudrillard, 2009, p. 154).

Este valor del cuerpo humano y particularmente del cuerpo femenino a partir de la sociedad de consumo, es otorgado a través de las exigencias del otro. Aprendemos a ser mujeres a partir de lo que se debe o no hacer con respecto al otro: no ser demasiado "insinua- da", "darse a respetar", no ser una "puta" o "no molestar al señor", son ideas o frases múltiples veces repetidas, incluso de mujer a mujer o de madre a hija. Aprendemos desde niñas a comportarnos de cierta manera $y / o$ avergonzarnos de actitudes fuera de estas normas.

De igual forma, los medios publicitarios desconocen la naturalidad de procesos biológicos en sus maneras de representarlos. Por ejemplo, todos los anuncios de toallas femeninas que tratan de probar la eficacia de su absorción, emplean un líquido azul para simular la sangre menstrual, imagen completamente alejada de la sangre menstrual real y su característico color. Estas falsas representaciones podrían favorecer la desnaturalización de procesos e incluso, miedos ligados a la noción del cuerpo femenino.

Estos miedos, junto con una sobre valoración a la juventud, han pautado el 'valor' del cuerpo de la mujer: el miedo a envejecer, a estar gorda, a dejar de ser atractiva, a no tener senos grandes, traseros voluptuosos, cintura definida, etcétera, son algunos miedos profundamente interiorizados por las mujeres y que son aprovechados por la industria para vender productos:

Los miedos del cuerpo son los que cuentan ac- 
tualmente con una mayor utilización por parte de las corporaciones, dado el enorme merca do que constituye todo lo relacionado con la imagen gracias al concepto de obsolencia percibida y a la sobrevaloración de la juventud que nos trasmiten los medios, miedo a la vejez, a estar gorda, a tener poco pecho, etc. En su versión más extrema los miedos del cuerpo pueden desembocar en Gerascofobia, o la fobia a la vejez; miedo extremo a las arrugas y al paso de los años que las mujeres enfrentan con cirugías plásticas, cremas y maquillajes caros que prometen lo imposible: detener el tiempo. Las mujeres más vulnerables a padecerla son aquellas que al sobrevalorar lo material y el logro de metas tienen serios problemas para enfrentar pérdidas como la belleza, la seducción y el dinero, entre otros (Cuenca, 2009, p. 4).

Luego entonces, el cuerpo femenino adquiere un valor que posteriormente se desvanece. Vale mientras sea joven y bello o mientras sea fértil, ya que una vez siendo madre, dicho cuerpo se vuelve asexual, se eleva a un nivel virginal y de pureza, el cuerpo de la madre intocable e inmaculada.

Esta valoración del cuerpo femenino, que como dijimos, sucede afuera del propio cuerpo, mantiene a muchas mujeres todavía frente a un desconocimiento del propio "código" de
Martha Irene Soria Guzmán su cuerpo, el cual no habitan ni reconocen como bello.

Según Naomi Wolf (1991), a pesar de la "liberación" femenina de los años setenta, muchas mujeres que tienen más dinero, poder y condiciones favorables dentro de su entorno profesional, siguen sintiéndose mal con respecto a sus cuerpos dejando ver una obscura veta de no aceptación a sí mismas:

...treinta y tres mil mujeres norteamericanas confesaron en las encuestas de una investigación que su meta más importante en la vida es perder entre 5 y 10 kilos. Muchas mujeres tienen más dinero, poder, campo de acción y reconocimiento legal del que jamás habíamos soñado, pero con respecto de cómo nos sentimos acerca de nosotras mismas físicamente, puede que estemos peor que nuestras abuelas no liberadas. Investigaciones recientes demuestran que las mujeres atractivas, exitosas y dueñas de sí mismas llevan una "sub-vida" secreta que envenena su libertad con ideas sobre la belleza. Es una veta oscura de odio hacía sí mismas, una obsesión con el físico, un terror de envejecer y un horror a la pérdida de control (Wolf, 1991, p. 215).

Estos problemas con la autoconcepción del cuerpo problematizan aún más la construcción de una femineidad partiendo del cen- 
tro o de la toma de decisiones de las propias mujeres, puesto que cuando alguna femineidad se construye lejos de los estereotipos, lo que no corresponde a estos estándares es juzgado, raro, diferente y descalificable.

Creer que el propio cuerpo deja de valer llegando a cierta edad, cuando se cumple la maternidad o ya no se es virgen; creer que no es bello porque no cubre los estándares de belleza establecidos por el "otro", supeditar la aceptación del cuerpo a la valoración de las masculinidades, alejarse del cuerpo y ver como ajenos los procesos propios del metabolismo femenino, avergonzarse de la menstruación y buscar ocultarla o no hablar de ella, pero sobre todo, no formar parte de la propia construcción de la femineidad y no ejercer el poder de decisión sobre lo que significa ser mujer; representan mecanismos de control basados en una violencia simbólica sumamente arraigada $y$ aceptada por la sociedad en su conjunto.

\section{CÓDIGO ABIERTO VS CÓDIGO CE- RRADO, DOS FORMAS DE ENTEN- DER LA TECNOLOGÍA Y LO FEME- NINO}

Vimos en la primera parte de este texto que la tecnología está en manos de ciertas corporaciones y que el software dentro de los gadgets es velado al usuario negándole la posibilidad de
Martha Irene Soria Guzmán saber cómo fue hecho, de dónde viene o saber si existe algún tipo de código malicioso que lo rastree o almacene sus actividades.

Por otro lado, hemos visto que la construcción de lo femenino desde finales del siglo XIX ha estado a cargo de una industria y que esta idea ha sido transmitida y aprendida culturalmente, replicando con ello actitudes que probablemente no permiten que muchas mujeres vivan en plenitud con su cuerpo.

Aunado a esto, y para abordar el eje tecnológico-femenino que propone este ensayo, se usará la aproximación que propone un estrecho vínculo del uso de los instrumentos y las armas, monopolizado por los hombres y el "uso" de los cuerpos femeninos, es decir, la hipótesis de que existe una relación estrecha entre los instrumentos usados tradicionalmente por ambos sexos y la división del trabajo y el dominio de los hombres sobre las mujeres (Tabet, 2005).

Paola Tabet sostiene a lo largo de un recorrido histórico sobre el uso de los instrumentos en su ensayo: Las manos, los instrumentos, las armas, que ancestralmente a las mujeres se les fue negada la posibilidad de extenderse más allá de sus propias fuerzas físicas, de la capacidad de sus manos, de prolongar su cuerpo y su brazo en instrumentos complejos que acrecienten su poder sobre la naturaleza: 
...la dimensión de este subequipamiento constante de la parte femenina de la especie humana y sobre su significado en la relación de dominio de los hombres sobre las mujeres, en la relación de apropiación material de las mujeres (Guillaumin 1978). Es necesario preguntarse en efecto qué ha significado y qué significa todavía hoy que un sexo haya tenido la posibilidad de extenderse más allá del propio cuerpo físico con instrumentos que amplían enormemente el poder sobre las cosas y sobre la sociedad y que en cambio el otro sexo esté limitado solamente a su cuerpo, a las operaciones hechas con las manos y por tanto a los instrumentos más simples de cada sociedad. Es necesario ver si ésta finalmente no es una condición necesaria para que las mujeres sean utilizables materialmente ellas mismas en el trabajo, en la reproducción, en la sexualidad (Tabet, 2005, p.67)

A pesar de que ambos sexos operan en un sistema de producción forjado desde la prehistoria, y que deviene en los últimos años en una macro estructura capitalista, hay una clara diferenciación con respecto a quiénes hacen determinadas actividades, con qué lo hacen y cómo lo hacen. Esto a su vez, se vincula con una dominación masculina:

El control por parte de los hombres de la
Martha Irene Soria Guzmán

producción y del uso de instrumentos y armas, se confirma como la condición necesaria de la dominación masculina sobre las mujeres: dominación basada en la violencia (monopolio masculino de las armas) y en el subequipamiento de las mujeres (monopolio masculino de los instrumentos). La exclusión de las mujeres de la fabricación y del uso de armas e instrumentos es la condición para usarlas en el trabajo, en la sexualidad, y en la reproducción de la especie (Tabet, 2005, p. 119).

A partir de lo anterior, no sería sorpresa entonces que los avances tecnológicos computacionales de la segunda mitad del siglo $\mathrm{XX}$, cuyos antecedentes se encuentran en los periodos de guerra, y particularmente en las armas de ataque, estén también monopolizados por los hombres. Así, tecnología y cuerpos femeninos son controlados eventualmente y en diversas épocas de la historia, por la figura masculina.

Exploremos otra coyuntura dentro del terreno tecnológico: la de los años setenta con el boom de la liberación que luego se ve mermado por el velo de un código cerrado. Por un lado, el software que en los setenta circulaba libremente como receta de cocina entre programadores y por otro lado la idea del cuerpo femenino que se libera a través de la píldora 
anticonceptiva y el uso de minifaldas. Sin embargo, esta súbita liberación está marcada por un culto a la belleza que sexualiza y convierte a la mujer en objeto que vende, al igual que el software que es vendido con su código fuente cerrado.

Dicho con otras palabras, la idea del cuerpo femenino es velado y asumido como prohibido, solo asequible para hombres que "poseen" una mujer, (maridos, amantes, padres). Con el boom de la publicidad que buscaba vender los excedentes de la producción industrial, el cuerpo femenino se "libera" y es mostrado sin ropa, vulnerable a la mirada masculina pero bajo los esquemas de la industria, cuyo objetivo principal es vender y que además le dice cómo hay que ser, cómo hay que actuar y cómo hay que vivir.

De la misma forma sucede con la tecnología y sus servicios en pleno siglo XXI, los cuales están casi al alcance de todos y a veces de forma gratuita (servicios como Facebook ${ }^{\circledR}$, Twitter ${ }^{\circledR}$ o Google () . El software parece haber sido liberado, pero lo cierto es que se encuentra bajo los esquemas de la industria que le dice qué hacer, a quién espiar y qué información recabar y a la que no le importa demasiado el usuario o consumidor final.

Así pues, el concepto de código cerrado puede observarse en el software privativo y en la femineidad, ya que por un lado los programas son un secreto, una especie de prohibición para los usuarios, de la misma forma en la que el cuerpo femenino es velado y prohibido. El alejamiento del propio cuerpo femenino impide que las mujeres se apropien de su femineidad, de la misma forma en la que el código cerrado no permite que nos apropiemos del software y de los aparatos que hace funcionar. Ambos se convierten en una caja negra.

Para ejemplificar esto, recuperaremos la imagen donde describíamos los comerciales que mostraban la eficacia de una toalla sanitaria con la absorción de un líquido azul. Éste código falso y alejado de la realidad, añade un velo al cuerpo femenino concibiendo a la menstruación como algo prohibido, sucio, tan vergonzante que es necesario disfrazar y ocultar.

Por último, cabe mencionar también que el software privativo se convierte en un producto para las masas logrando confundirse con el propio equipo de cómputo (que todas las PC de escritorio tengan instalado de fábrica determinado sistema operativo sin darle cabida a la libertad que el usuario tiene de elegir el que más le convenga). De igual forma, la falsa idea de la femineidad se convierte en un producto de masas confundiendo la representación de 
la mujer de los medios con la mujer misma: todas deben ser de tal o cual manera y con ello responder a estereotipos.

Sin embargo, una contra propuesta al control tecnológico mediado por las corporaciones, podría ser entender los cuerpos y la tecnología desde la perspectiva del código abierto, donde los "saber hacer" están al descubierto y donde cualquier persona que pueda leerlos o estudiarlos, también pueda modificarlos; esto es, volviendo a la metáfora de la receta de cocina, la posibilidad de saber los ingredientes y los pasos para la elaboración nos permitirá crear nuestra propia tecnología y nuestra propia idea sobre nuestros cuerpos.

Si logramos develar la manera en la que los medios codifican los mensajes, si conocemos la verdad de nuestros cuerpos y basamos nuestro acercamiento a ellos en una estructura de apertura y lenguaje directo, será, eventualmente más sencillo apropiarnos de nuestra femineidad y también de la tecnología que usamos.

\section{EL CAMINO DEL CONOCIMIENTO ABIERTO: EL PARADIGMA DEL SOFTWARE LIBRE}

Como ya dijimos, se propone acercarnos a la filosofía del software libre, que hasta ahora, sabemos fomenta la compartición del cono-
Martha Irene Soria Guzmán cimiento a través de la apertura del código. Sin embargo, su historia y fundamentos nos ofrecen también muchas similitudes con algunos feminismos.

En 1984, el fundador de la Free Software Fundation, Richard Stallman se fijó firmemente defender la práctica del intercambio y la "libertad" de los usuarios del software y comenzó a escribir un sistema operativo que denominó GNU, cuya característica principal era que cada parte que lo compone y lo hace funcionar fuera realizado por una comunidad de programadores que lo mejorara y compartiera, así cada persona que tuviera acceso a él podría modificarlo y adaptarlo según sus necesidades y plataforma que se utilizara, aspirando así a crear el sistema operativo perfecto a través de un principio básico de compartición del conocimiento (GNU, s.f.) y lejos de los acuerdos de confidencialidad.

Paulatinamente, el proyecto GNU causó una transmisión acelerada de información por compartición, ya que varios programadores decidieron contribuir con él. Fue en 1991 cuando el finlandés Linus Torvalds programó el núcleo y la parte fundamental para el sistema operativo GNU, el kernel, que fue llamado Linux, basándose en un sistema operativo con fines didácticos, Minix. Hoy día, 
Linux es uno de los programas libres más utilizados para el sistema operativo GNU (McAllister, 2006). A partir de que Linux es liberado, dicho sistema operativo empezó a evolucionar rápidamente debido a que muchos programadores han contribuido a su desarrollo y mejora, lo que lo convierte en uno de los sistemas operativos más estables.

Con GNU y Linux juntos, comienza uno de los crecimientos más importantes del software libre, la incursión en el mundo empresarial, ya que algunas empresas comenzaron a basar su estrategia de negocios en hacer consultorías, distribución y servicios alrededor de este nuevo y creciente sistema operativo. La estrategia fue comenzar a vender "misterios" alrededor del software libre los cuales podían ser resueltos por quién supiera de lenguaje de programación y no "secretos" indescifrables que una sola empresa conociera. No se trata de vender el secreto del código, sino la libertad del servicio.

Con estos antecedentes, podemos hacercarnos a una definición formal del software libre, cuya ideología está basada en el concepto de "libertad", tal como se explica a continuación.

\section{DEFINICIÓN DE SOFTWARE LIBRE}

El término software libre o free software, como ya dijimos, se refiere a aquellos programas cuyo
Martha Irene Soria Guzmán código está abierto para que cualquier usuario pueda modificarlo, usarlo, estudiarlo $\mathrm{y} / \mathrm{o}$ distribuirlo sin que esto represente un problema legal.

También ya dijimos que el término "código abierto" o en inglés, open source, se refiere a que las líneas de texto en las cuales están escritas las instrucciones que sigue la computadora para ejecutar dicho programa, están disponibles para que todos los usuarios puedan conocerlas y así, mejorar o adaptar dicho código según sus necesidades. Sin embargo, el software libre no se trata de una copia no autorizada ni mucho menos de aquel descargable desde internet de manera gratuita, el llamado freeware. El software libre debe poseer la autorización para utilizar un código abierto. Free software y open source son términos distintos pero complementarios.

El software libre con sus líneas de código abierto, representa la libertad de que cualquiera puede hurgar en él, lo cual a la larga permitiría que el programa sea modificado e incluso mejorado a una velocidad mucho más eficiente que el desarrollo del software privativo, dando como resultado la creación de un mejor software con la colaboración de muchas personas a la vez, tal como lo explica Raymond en La catedral y el bazar en lo que él llama la ley Linus: 
Dada una base lo suficientemente amplia de probadores y colaboradores, casi todos los problemas se identificarán con rapidez y su solución será obvia para alguien. O expresado con menor formalidad: "con un número de ojos suficiente, todos los errores son irrelevantes" (Raymond, 1997, p. 16).

Ahora bien, el término free software fue acuñado en ingles, por lo que, puede existir una confusión en dicho idioma ya que la palabra free se refiere a "libre" y al mismo tiempo al concepto de "gratis", es por eso que la Free Sofware Fundation, aclara que "el software libre es una cuestión de libertad, no de precio. Para entender el concepto, debería pensar en libre como en libre expresión, no como en barra libre" (Stallman 2004).

Es por eso que el software libre puede estar disponible para su distribución comercial sin que eso le quite su calidad del "libre", ya que se pudo pagar alguna cantidad para obtener una copia del software para cubrir algunos costos de distribución o bien, obtenerlo sin costo alguno, ambas opciones no afectan que un software siga siendo libre o no, siempre y cuando se cumplan las cuatro libertades.

Estas cuatro libertades son a su vez los cuatro principios del software libre, son cuatro libertades que tienen los usuarios del software y
Martha Irene Soria Guzmán que necesariamente deben de ser cumplidas para que se le considere como tal. Dichas libertades fueron acuñadas por el propio Richard Stallman y posteriormente se convirtieron en bandera de la Free Software Fundation:

Libertad 0. Libertad de usar el programa para cualquier propósito.

Libertad 1. Libertad de estudiar el funcionamiento del programa y adaptarlo a las necesidades. El acceso al código fuente es una condición previa para esto.

Libertad 2. Libertad de redistribuir copias, y con ello poder ayudar al prójimo.

Libertad 3. La libertad de distribuir copias de sus versiones modificadas a terceros.

Según estas ideas, los programas de computo no deben tener un "dueño" que se reserve los derechos, y eso no significará que no sea "autor" del mismo. ${ }^{5}$ Se basa en la premisa de que el "conocimiento es de todos", pues al compartir dicho conocimiento ocurre un beneficio, no solo particular, sino a nivel comunitario. Al mejorar un software con la

${ }^{5} \mathrm{El}$ concepto de "autor o autora" separado del de "dueño o dueña". Donde las personas que tienen la autoría no pierden el crédito por la obra pero tampoco se "adueñan" de ella para restringir su posterior uso, mejora, estudio y distribución. 
ayuda de una comunidad, esa misma comunidad se beneficia con las mejoras.

El abogado estadounidense, Lawrence Lessig, se inspira en la filosofía del software libre y acuña recientemente el concepto de cultura libre, la cual define la visión de una cultura basada en la libertad de distribuir y modificar trabajos y obras creativas: "Las culturas libres son culturas que dejan una gran parte abierta a los demás para que se basen en ella, las que no son libres, las culturas del permiso, dejan mucho menos" (Lessig, 2005). Se refiere a basarse en lo que han hecho otros, en lo que se ha hecho en el pasado, en una cultura basada en la compartición y distribución libre del conocimiento.

El conocimiento colaborativo es otro de los pilares del software libre, esto es que entre más personas colaboren para la creación de un software, se creará un mejor programa a una velocidad mucho mayor y los usuarios se verán beneficiados con ello y parte del principio que "la compartición con los demás constituye la base de la sociedad" (Stallman, 2004, p. 18).

Resumiendo las ideas planteadas en este apartado, el software libre se basa en la cultura libre, conocimiento colaborativo, comprartición de conocimiento, libertad en el conocimiento, copyleft y sobre todo, libertad.
Martha Irene Soria Guzmán

\section{SOFTWARE LIBRE Y FEMINISMOS: HACKEAR EL SISTEMA}

La filosofía del software libre, desde su perspectiva del código abierto, ofrece un paralelismo con el feminismo en tanto que la noción de género y programas de cómputo son normativa e institucionalmente reguladas, tan como lo dice Pujol y Montenegro:

Free software gives users the freedom to share, study, modify and distribute the code and, therefore, constitutes a techno-social ordering in which semiotic (i.e., the appeal to "freedom") and material (i.e., the possibility of code modification) elements conform to a particular configuration. Because gender is also a semiotic-material ordering, a parallelism between gender and software can be established in the sense that both are normatively and institutionally regulated. This unisonance establishes a common ground for transforming the techno-gender assemblage (Pujol y Montenegro, 2015, p. 181).

Al igual que el feminismo, el software libre subyace una propuesta subversiva que cuestiona y desestabiliza la relación de las normas legales y económicas que han definido y legitimado la producción y distribución sesgada del conocimiento, posibilitando con ello la activación de una apropiación tecnológica por parte de la comunidad que la utilice. 
The regulatory mutation created by open-source licenses allows for the circulation of technologically-mediated content (Scolari 2009) and, like gender performances, it has a subversive effect since it destabilizes the legal-economic norm that defines and legitimates certain subject positions in the production and distribution of knowledge and cultural productions. The techno-feminist assemblage is built upon the possibility of active technological appropriation by participants. These networks are models of effective action against both consumerism and technological passivity, and seek the re-appropriation of the hegemonic mechanisms of cultural production in order to create a new technological grammar (Pujol y Montenegro, 2015, p. 181).

Además de lo anterior, la filosofía del software libre tiene un vínculo importante con la cultura hacker, donde un hacker es quien lleva a cabo soluciones de manera creativa y diferente a la esperada en aras del cambio social. Bajo este concepto, cualquier persona dentro de una lucha social en miras de mejorar el mundo en el que vive, es sin duda un hacker, por lo tanto, las feministas son, en sí mismas, hackers del sistema.

Nosotros hackeamos la abstracción. Producimos nuevos conceptos, nuevas percepciones, nuevas sensaciones hackeadas a partir de datos en bruto. Sea cual sea el código que hackeemos, ya sea lenguaje de programación, lenguaje poético, mate-
Martha Irene Soria Guzmán

máticas o música, curvas o colores, somos nosotros quienes abstraemos nuevos mundos (Wark, 2006, p. 16).

Esta cita podría ser proclamada en algún manifiesto feminista, donde son las propias mujeres las que abstraen nuevos mundos posibles. Para ello, es necesario hackear desde adentro el sistema que ha forjado muchas de nuestras ideas preconcebidas de nuestros cuerpos, incluso backeando nuestros propios cuerpos, decidiendo por ejemplo, si queremos o no ser madres sin que eso signifique una automática descalificación. Hackear es diferir (Wark, 2006)

En su libro Un manifiesto hacker, MacKenzie Wark (2006) habla de cómo las representaciones se convierten en medios de opresión por sí mismas. El autor advierte que la cultura backer rechaza la representación misma, o bien, fomenta mantener el control del significado de la propia representación, controlando también la adhesión de sus miembros a ella. Esto podría ser otro vínculo importante con la lucha feminista, ya que sería posible rechazar cualquier representación de la figura femenina que estuviese fuera del propio control de la mujer, posibilitando que sea cada una la que "invente" su propia representación y controle su propia significación. 
Por último, no hay que olvidar la aproximación desde el constructivismo tecnológico, que si bien no fue uno de los ejes de este ensayo, sí ofrece otra línea argumental que refuerza la idea de que cada persona va a encontrar su propia manera de asumir, usar y apropiarse de la tecnología, principio sine qua non de la figura del hacker. El término "flexibilidad interpretativa" ofrece una dimensión social de la tecnología a través de la deconstrucción del artefacto, en este caso, el objeto computacional. Para Wieber Bijker (1995), uno de los autores del término, esta flexibilidad interpretativa se manifiesta en la medida en la que se consensúan, negocian y discuten los usos y significados de un artefacto, proceso necesario para una posible apropiación tecnológica.

\section{CONCLUSIONES}

Aún quedan algunos vacíos que valdría la pena explorar en otros textos al respecto, como por ejemplo el hecho de que a pesar de los vínculos, aún son pocas las mujeres dentro de la propia comunidad de software libre, como menciona Haché, Cruels y Vergés en su proyecto de investigación cyberfeminista, acerca de la presencia de mujeres en el desarrollo de la informática así como en el software libre:

...entre 100 programadores, uno se hace hacker, $\mathrm{y}$
Martha Irene Soria Guzmán

de 100 programadores encontramos solo a 10 mujeres, ¿cuántas mujeres programadoras necesitamos para encontrar una mujer hacker? Estas suelen elegir con mucho menos frecuencia el desarrollar una formación y carrera como programadoras y/o informáticas (Haché, Cruels y Vergés. 2011, p. 2).

De la misma forma, las similitudes entre cuerpo femenino y software/tecnología que propone el presente texto, aún están desdibujadas y hay que considerarlo solo como un ligero acercamiento, porque cabe la posibilidad de que estas similitudes puedan observarse con mayor claridad desde dentro de los corporativos y desde las reglas del sistema mediático.

Por lo pronto, dentro del contexto de dominación y control a través de la mediación, la propuesta es conocer nuestro cuerpo lejos de estereotipos impuestos, de la misma forma en la que debemos conocer la tecnología que usamos e idealmente crear la propia; lejos del escrutinio de las corporaciones y la idea de estatus que promueven los gadgets en del consumo de masas.

Para ambos casos, se propone la metáfora de acceder a la caja negra, al código de los cuerpos femeninos tanto como al código fuente del software, el cual depende del cono- 
cimiento libre y de un saber hacer que pocas veces o casi nunca, se comparte en la industria. Se propone la imagen del código abierto como metáfora de las ideas libres y modificables en comunidad.

Por ello, saber el por qué y para qué nos presentan algunas publicidades, conocer el código oculto detrás de la comunicación que transmite estereotipos o falsas ideas de lo femenino, así como develar la intención de control corporativo en las representaciones del cuerpo de la mujer, nos permitiría detener un poco la dominación que proviene de "fuera" y decidir por nosotras mismas de qué manera asumimos nuestro cuerpo y nuestra femineidad y con ello, hackear el sistema. Los femi- nismos, como muchas otras luchas sociales, podrían asumirse como hackers del sistema.

El feminismo y el software libre convergen en el trabajo comunitario, partiendo de la idea de que sólo en comunidad podrá ser posible, por un lado, la liberación real del cuerpo femenino y por el otro, la libertad del conocimiento, ambos bajo el yugo de la industria.

Por último, aunque no menos importante, el software libre se convierte en un arma para protegernos de la vigilancia masiva que está en ciernes y que hoy ya no es un secreto. Solo el software libre es una herramienta tecnológica viable para la emancipación de corporaciones e industria que lucran con nuestros datos, nuestros miedos y nuestros cuerpos. 


\section{FUENTES CONSULTADAS}

Ascención, A., (2016). “3,763 mdp en tablets y laptops, gasto cuestionado a la SEP”. En: CNNExpansion.com. www.cnnexpansion.com/economia/2015/12/21/3763-mdp-en-tablets-y-laptopsun-gasto-cuestionado.

Baudillard, J. (2009). La Sociedad de consumo, sus mitos y sus estructuras. Madrid: Siglo XXI de España Editores, $255 \mathrm{pp}$.

Bernard, E. (2015). "La utilización de la imagen femenina de manera erótica o sexualizada en la publicidad convencional y en la publicidad online". En: La Pantalla Insomne, 2da. edición, ampliada. Universidad de la Laguna.

Bijker, W. E. (1997). Of bicycles, bakelites and bulbs: toward a theory of sociotechnical change. MIT Press. Recuperado a partir de https://books.google.com.mx/books?id=IsbmwN8-m1cC.

Bordeu. P., (1998). La dominación masculina. Barcelona, España: Editorial Anagrama.

Busaniche, B. y Heiz, F. (2011). Cartillas de introducción al software libre. Proyecto Nomade. Ed. Lila Pagola. Disponible en www.nomade.org.ar/sitio/?page_id=104.

Castañeda, L.I. (2013). "El celular como elemento de la identidad juvenil". En: Revista de Educación y Desarrollo, 13, abril-junio.

Chazin, S. (2011). Marketing Apple. The Marketing Apple, eBook. Obtenido de www.marketingapple.com/marketing_apple/the-marketingapple-ebook.html.

Cuenca, M. (2009). "El miedo al servicio del consumo femenino". En: Red Visual, núm. 10, mayo 2009.

Foucault, M. (1974). Vigilar y castigar: "nacimiento de la prisión". Buenos Aires, Argentina: Siglo Veintiuno Editores Argentina.

GNU Operating Systems (s.f.). "Visión general del proyecto GNU, Historia del proyecto GNU. Estados Unidos”. GNU Project, Free Software Fundation. Recuperado de www.gnu.org/gnu/gnuhistory.es.html.

Haché, A., Cruels E. y Vergés, N. (2011). Mujeres programadoras y mujeres hackers. Una aproximación des de Lela Coders. En: Rebelion.org.

Lesing, L. Cultura libre, Madrid: Traficantes de Sueños.

Mathieu, N. G. (2005). “¿Identidad sexual/sexuada/ de sexo? Tres modos de conceptualización de 
la relación entre sexo y género". En Curiel O. y Falquet J. (ed.). El patriarcado al desnudo: tres feministas materialistas, 130-175. Buenos Aires, Argentina: Brecha Lésbica.

Morán, P. (2006). "Si queremos asegurar algún logro social, como por ejemplo, la salud pública o el medio ambiente, siempre necesitaremos el software libre". España: Eroski Consumer. Disponible en www.consumer.es/web/es/tecnologia/software/2006/03/17/150227.php.

Notimex (2014). "SEP entregará más de 700 mil tablets a alumnos y maestros". En: Milenio.com. Obtenido de www.milenio.com/politica/sep_regala_tablnets-tabletas_y_sep-tablets_y_maestrostablets_sep_y_alumnos_0_352764943.html

Pujol, J. y Montenegro, M. (2015). "Technology and feminism: a strange Couple”. En: Tecnología y feminismo: una extraña pareja, (51), 173-185. Disponible en https://doi.org/10.7440/res51.2015.13.

Raymond, E. (1997). La catedral y el bazar. Argentina: Openbiz.

Ricaurte, P., Nájera, J. y Robles, J. (FALTA EL AÑO). "Sociedades de control: tecnovigilancia de Estado y resistencia civil en México”. En: Revista Teknocultura, vol. 11, núm. 2, 259-282.

Rodríguez, D. (2011). "Por qué nos vuelve locos Steve Jobs sobre un escenario. Trending topics". Blog de El Pais. Obtenido de http://blogs.elpais.com/trending-topics/2011/03/por-que-nosvuelve-locos-steve-jobs-sobre-un-escenario.html.

Simmel, G. (1934). Cultura femenina. Madrid: Revista de Occidente, 329 pp.

Soria, M. (2012). Software libre, herramienta alternativa para la creación visual digital. España: Editorial Académica Española.

Stallman, R. (2004). Software libre para una comunidad libre. Madrid: Traficantes de Sueños.

Tabet, P. (2005). “Las manos, los instrumentos y las armas”. En: Curiel O. y Falquet J. (ed.). El patriarcado al desnudo: tres feministas materialistas, 130-175. Buenos Aires, Argentina: Brecha Lésbica.

Villanueva, G. (2010). "E-competencias: nuevas habilidades del estudiante en la era de la educación, la globalidad y la generación de conocimiento". En: Signo y Pensamiento, vol. XXIX, núm. 56, enero-junio, 124-138. Bogotá, Colombia: Pontificia Universidad Javeriana.

Wolf, N. (1991). El mito de la belleza. Barcelona: Emecé Editores. 